\title{
ESTUDiO EXPLORATORIO DE LA RED DE PREFIJOS EN ESPAÑOL
}

\author{
ANTONIO RIFÓN \\ Universidade de Vigo
}

Title: Exploratory Research on Prefixal Network in Spanish.

\begin{abstract}
The goal of this paper is to do an exploratory research of the network of the spanish prefixes. First, we analyze and compare the classifications done by three bibliographical sources to establish a semantic classification of the prefixes; secondly, we will create a graph not directed and weighted in that the nodes are the prefixes and the edges, the shared meanings. Hereby, the prefix A will be related to the B if they share meant, that is to say, if it has meant that they can create both prefixes. We will analyze the global measures of this graph and his subgraphs focusing on his density, modularity and degree of clustering, we will establish his communities and will study two measures of centrality, the closeness and the betweenness centrality.
\end{abstract}

Key words: Morphology. Word Formation. Prefixes. Network. Graphs. Centrality Measures.

\section{INTRODUCCIÓN}

En el CILX-20 8 (Rifón: 20 i 8), he presentado una comunicación en la que se hacía un análisis exploratorio de la red semántica de los prefijos; en esta red, los nodos eran los significados de los prefijos y las aristas, los prefijos compartidos. De esta manera, dos significados estaban relacionados si podían ser creados por algún afijo común y el grado de la relación dependía del número de prefijos compartidos.

En esta ocasión completaré ese estudio exploratorio con el análisis de la red de los prefijos, en la que los nodos son los prefijos y las aristas los significados que comparten. De esta manera, dos prefijos estarán relacionados si pueden ser empleados para crear el mismo significado (entrelinear, interlinear).

El objetivo general del artículo es analizar las posibilidades que tiene la aplicación de la teoría de grafos o redes al estudio de la red de prefijos constituida por las relaciones de semejanza léxica o sinonimia.

Partiremos de dos ideas básicas: los prefijos son polisémicos o polifuncionales y existen relaciones de sinonimia entre prefijos. La primera idea 
no deja de ser controvertida en la teoría morfológica (vid. Rainer, Dressler, Gardani, y Luschützky: 20I4), pero, en este momento, no la discutiremos; un prefijo puede crear derivados con diferentes significados y petenecer a distintas familias funcionales desde el punto de vista semántico. Por ejemplo, super-puede emplearse para formar derivados con significados espaciales (superíndice, superlingual) e intensivos (superarrastrero, superbarato).

La segunda idea es también problemática por dos razones. La primera, heredada de la semántica, se debe al concepto de sinonimia, que es, en sí mismo problemático, por eso emplearé, en algunos casos, similiraridad. La segunda, derivada de la descripción morfológica, pues esta intenta mantener la relación uno-a-uno y tiende a reconocer matices de significado diferente si el afijo es diferente. Para nuestro caso, obviaremos ambas dificultades y reconoceremos que, en muchos casos, las posibilidades funcionales de los prefijos se solapan y distintos prefijos sirven para crear derivados con el mismo significado morfológico; por ejemplo, super-y sobre- pueden emplearse para crear derivados deadjetivales que indiquen la posesión de un grado alto de una cualidad (supercaro / sobrecaro).

Además de las redes semánticas reconocidas aquí, hay muchos otros tipos de estructuras u organizaciones morfológicas; entre ellas, no se pueden olvidar las ya clásicas familias léxicas (p. e. Pena Seijas y Campos Souto: 2009), los, muy de moda, paradigmas derivativos con sus diferentes enfoques (Guilbert: 1975; Marle: 1985; Bauer: 1996; Blevins: 200 I; Beecher: 2004; Štekauer: 2014; Subbotina, Fatkhutdinova, y Ratsiburskaya: 20ı7; Bonami y Strnadová: 2018) o la aplicación de los frames a la formación de palabras (Plag, Andreou y Kawaletz: 2017).

La teoría de redes se ha aplicado a diversas áreas relacionadas con el lenguaje, sobre todo, en lo que se refiere al PLN para la cofección de resúmenes y la extracción de frases o palabras clave (vid. Mihalcea y Radev: 2OI I; Lahiri: 2OI 3), pero también a aspectos descriptivos del lenguaje tanto 
léxicos como gramaticales (p. e. Ferrer I Cancho y Solé: 200 I; Biemann, Choudhury y Mukherjee: 2009), aunque son pocos los estudios que aplican directamente esta teoría al análisis de la morfología derivativa (Baayen: 20 o; Rifón: 2016).

Dicho esto, se puede, entonces, establecer una relación entre los prefijos que pertenecen a la misma familia funcional, es decir, que crean derivados con el mismo significado morfológico y, por tanto, se puede construir una red en la que los prefijos sean los nodos y los significados compartidos los ejes. Nuestra intención es construir esa red y analizarla a partir de las herramientas y cálculos de la teoría de redes.

2. Métodos y materiales

El método seguido para obtener los datos objeto del análisis se ha desarrollado en cuatro fases:

I. Recopilación de los significados morfológicos.

2. Comparación de las clasificaciones.

3. Confección de una clasificación y asignación de significados.

4. Construcción del grafo

\section{I. Recopilación de significados}

Establecer los significados morfológicos de los prefijos no es una tarea fácil. El primer problema deriva de las múltiples clasificaciones hechas y de las también variadas terminologías empleadas. El segundo, de la dificultad para establecer el límite entre el significado propiamente morfológico, el aportado por el prefijo o la pauta de derivación, y el puramente léxico, propio del derivado individual.

Nos propusimos, en vez de abordar directamente la creación de una nueva clasificación que se añadiese a las muchas hechas, recopilar aquellas que se pueden considerar fundamentales, para, una vez comparadas, proponer una que las abarque y que trate de ser, en la medida de lo posible, fiel 
a la terminología empleada en ellas. Escogimos tres clasificaciones: Rainer (1 993), Varela Ortega y Martín garcía (1999) y Real Academia Española y Asociación de Academias de la Lengua Española (2009).

Consideramos que estas tres obras recopilan, en parte, los trabajos de otros autores sobre la prefijación y, además, tratan la prefijación de forma global, es decir, estudian todos los prefijos y todos los significados. Estas dos características hacen de ellas fuentes suficientes para el trabajo exploratorio que nos hemos propuesto.

Las clasificaciones y significados se recogieron en una base de datos que relacionaba cada prefijo con su clasificación, autor y ejemplos, esta base permitía una visión global y relacional de las tres clasificaciones.

\subsection{Comparación de las clasificaciones}

En esta fase se atendió a las diferencias y semejanzas entre las clasificaciones con el fin de recuperar las primeras y resolver las segundas. Para ello, se confeccionaron tablas de comparación como la de la Tabla i en la que se comparan los significados que podríamos llamar gradativos establecidos por Varela Ortega y Martín García (1 999) y Real Academia Española y Asociación de Academias de la Lengua Española (2009):

\begin{tabular}{|l|l|}
\hline Real Academia et alii. & Varela Ortega y Martín García \\
\hline Gradativo: Intensidad: Exceso & Exceso \\
\hline Gradativo: Intensidad: Grado máximo & Gradativo: Cualidad: Positivo: Máximo \\
\hline & Superlativo \\
\hline Gradativo: Intensidad: Grado alto & Gradativo: Cualidad: Positivo: Medio \\
\hline Gradativo: Intensidad: Grado medio & Gradativo: Cualidad: Negativo: medio \\
\hline Gradativo: Intensidad: Grado escaso o insuficiente & Gradativo: Cualidad: Negativo: Grado cero \\
\hline & Gradativo: Tamaño o cantidad: Exceso \\
\hline Tamaño: Grande & Gradativo: Tamaño o cantidad: Positiva \\
\hline Tamaño: Pequeño & Gradativo: Tamaño o cantidad: Negativa \\
\hline Gradativo: aproximativo & \\
\hline
\end{tabular}

Tabla ı: Correlaciones entre significados gradativos

Esta tabla es solo una muestra de algunas dificultades que surgen cuando se comparan las clasificaciones con el fin de unificarlas, pero hay muchas más. El problema de fondo es que, si bien las tres clasificaciones son muy cercanas, no son, ni mucho menos, semejantes, lo que hace que 
aparezcan innumerables problemas de unificación e incluso ciertas contradicciones.

El caso del prefijo con- sirve para mostrar las principales dificultades. Rainer (1993: 317) señala que con verbos indica "gemeinsam mit"("junto con"), mientras que Varela Ortega y Martín garcía ( I999: 5016) lo clasifican dentro de los locacionales o comitativos, indicando que es solo productivo con valor comitativo; a esto se añade que Real Academia Española y Asociación de Academias de la Lengua Española (2009: 704) lo clasifican dentro de los prefijos de incidencia argumental dando lugar a "predicados colectivos o simétricos". Al final la pregunta permanece ¿pero qué valor tiene este prefijo? En este caso se presentan, entonces dos problemas: la variabilidad terminológica y las diferencias de clasificación.

La terminología empleada por los distintos autores no es la misma, pero, esta variabilidad no se da solo interauores, sino también dentro de un mismo autor. Por ejemplo, Real Academia Española y Asociación de Academias de la Lengua Española (2009: 670, 684) cuando hace una tabla con los significados prefijales habla de Espaciales, pero, cuando los trata, el apartado se titula Prefijos de sentido locativo. La variación terminológica interautores e intraautor provoca un alto grado de inexactitud, pues, en muchos casos, cuando se emplean dos etiquetas diferentes no es posible saber si estas se aplican al mismo concepto o hay diferencias entre ellas.

Las diferencias entre las clasificaciones no solo se deben a cuestiones terminológicas, sino también están provocadas por variaciones en las propiedades empleadas para hacer las clasificaciones. Por ejemplo, la Real Academia Española y Asociación de Academias de la Lengua Española (2009: 725) clasifica los prefijos anti- y pro- entre, respectivamente, los opositivos o de actitud favorable; mientras que Varela Ortega y Martín garcía (1999) incluyen al primero en los opositivos junto con los negativos, categorías que los anteriores autores mantenían separadas, y a pro- entre los de posición.

En otra ocasión, la Real Academia Española y Asociación de Academias de la Lengua Española (2009) reconoce un significado que podemos etiquetar como Adjetival: Tamaño: Grande para supernova, mientras que Va- 
rela Ortega y Martín García (1999) emplean algo así como Gradativo: Tamaño o cantidad: Positiva, por lo que parece que el rasgo adjetival no juega el mismo papel en ambas clasificaciones.

La variación terminológica es un problema serio en cualquier disciplina científica y, aquí, no parece que estemos exentos de ella; si a esto añadimos el hecho de que las propiedades clasificatorias son variables, las clasificaciones se hacen, a pesar de ser similares, difícilmente conmensurables. Aun así, hemos tratado de unificarlas y hacer una clasificación de la fusión de las tres con el único objetivo de que sea útil para nuestro estudio.

\subsection{Confección de una clasificación y asignación de significados}

La nueva clasificación trata de recoger los significados reconocidos por los autores y, a la vez, resolver sus discrepancias, pero, al tratarse de un estudio exploratorio, no pretende ser definitiva limitando su alcance a dos niveles de clasificación.

En primer lugar, no es definitiva porque el método empleado no nos asegura que todos los significados existentes estén recogidos en la clasificación; para ello, no solo deberíamos extender nuestras fuentes, sino, también analizar, a partir de un corpus, los derivados creados por cada prefijo para, de esta manera, estar seguros de forma más fiable que todos los significados existentes están recogidos.

En segundo lugar, su alcance es limitado pues el método no nos permite llegar a niveles más bajos de diferenciación; así, por ejemplo, se ha distinguido un significado gradativo: mucho /alto pero no se ha diferenciado dentro de él si graduaba cualidad o tamaño, diferencia que aparece cuando se trata, por ejemplo, el prefijo macro- cuyos derivados usados como ejemplos suelen indicar gradación de tamaño o cantidad, pero también podemos documentar casos de cualidad, macrobueno.

Además, es difícil determinar, de una vez por todas, los posibles significados de tercer nivel; unas veces por cuestiones de documentación, como en el caso anterior de macrobueno; otras por cuestiones semánticas y morfológicas. En el significado espacial: alrededor de tenemos casos que indican movimiento, circunnavegar, casos que solo indican posición, circunyacen- 
te, pero casos que puede indicar ambos, circunsolar; movimiento y posición, que podrían ser considerados significados del tercer nivel del significado espacial, parecen derivar de contextos morfológicos, el tipo de base, y semántico-contextuales.

A pesar de todos estos problemas, se han hecho algunos reajustes con respecto a las clasificaciones estudiadas, unos de carácter puramente terminológico; por ejemplo, como se ve en la Tabla I, un autor habla de grado máximo y alto y las otras autoras, de grado máximo y medio. Esta unificación terminológica es relativamente sencilla, solo hay que escoger el término más adecuado que mantenga la coherencia de la clasificación final.

Pero, en algunos casos, la reclasificación ha ido más allá; por ejemplo, el prefijo meta- ha sido clasificado principalmente como un locativo: más allá o posición sobrepasada; sin embargo, si bien este significado puede ser aplicado históricamente a un término como metafísica, no parece que pueda ser aplicado en la actualidad a derivados como metamatemática o metalenguaje en los que, el significado de meta- parece más cercano al de auto-, reflexivo, que al espacial de ultra- o pos-.

\subsection{Confección del grafo}

Queda ahora confeccionar el grafo en el que los nodos o vértices son los prefijos entre los que se establecen aristas o ejes si crean palabras con el mismo significado morfológico.

Si el prefijo A comparte algún significado con el B, se establece una arista que relaciona $\mathrm{A}$ y $\mathrm{B}$; esta arista une $\mathrm{A}$ y $\mathrm{B}$ en ambas direcciones, tanto de $\mathrm{A}$ a $\mathrm{B}$ como de $\mathrm{B}$ a A; pero, para simplificar las relaciones y al ser la arista de doble dirección, hemos considerado que la relación es simétrica, es decir, podemos unificar ambas aristas en una no dirigida para obtener un grafo no dirigido.

Tanto los nodos como las aristas no dirigidas han sido pesadas o ponderadas. El peso de los nodos es igual al número de significados que el prefijo puede crear. Si el prefijo A crea 5 significados diferentes, el nodo tendrá un peso de 5 . 
Para establecer el peso de la arista entre dos nodos se ha hecho un promedio entre los significados que relacionan ambos prefijos y los que podrían relacionarlos. Por ejemplo, para ponderar la arista que une los prefijos A y $B$, se ha de tener en cuenta el número de significados comunes y se halla, para cada prefijo, la probabilidad de que aparezcan esos significados teniendo en cuenta el número total de significados; así, si A y B tienen 3 significados comunes, y A tiene 6 significados en total y B, 4, el prefijo A aportará a la relación un peso de $3 / 6=0,5$ y el $\mathrm{B}, 3 / 4=0,75$. Para hallar el peso total de la arista se calcula el valor medio del peso aportado por cada prefijo $(0,5+0,75) / 2=0,625$.

De esta manera el prefijo mega- que puede crear 2 significados (Intensivo: Gradativo: Mucho /Alto, Cuantificativo: Multiplicativo) y el archi- que puede crear 4 (Intensivo: Gradativo: Mucho /Alto, Jerarquí: Superior: Rango, Jerarquia: Superior, Intensivo: Gradativo: Demasiado / Excesivo) tendrán esos valores como peso del nodo y el de la arista será, ya que tienen i significado común (Intensivo: Gradativo: Mucho /Alto), la suma del peso que aporta mega-, $1 / 2=0,5$, y del que aporta archi-, $1 / 4=0,25$, dividida entre 2, por lo que el peso total será $(0,5+0,22) / 2=0,38$.

3. Resultados y discusión

El grafo obtenido (Figura I) tiene las siguientes características globales (Tabla 2):

\begin{tabular}{|l|r|}
\hline \multicolumn{2}{|c|}{ Medidas globales del grafo } \\
\hline $\mathrm{N}$ (nodos-prefijos) & 66 \\
\hline $\mathrm{L}$ (aristas-relaciones) & 246 \\
\hline $\mathrm{d}$ (diámetro) & 5 \\
\hline $\mathrm{D}$ (Densidad) & $0, \mathrm{I}$ 5 \\
\hline Modularidad & 0,602 \\
\hline Componentes conexos & 7 \\
\hline$l_{G}$ (Longitud media del camino) & $2,4 \mathrm{O}$ I \\
\hline $\bar{C}$ (Coeficiente medio de clustering) & $0,8 \mathrm{O} 2$ \\
\hline
\end{tabular}

Tabla 2: Medidas globales del grafo 


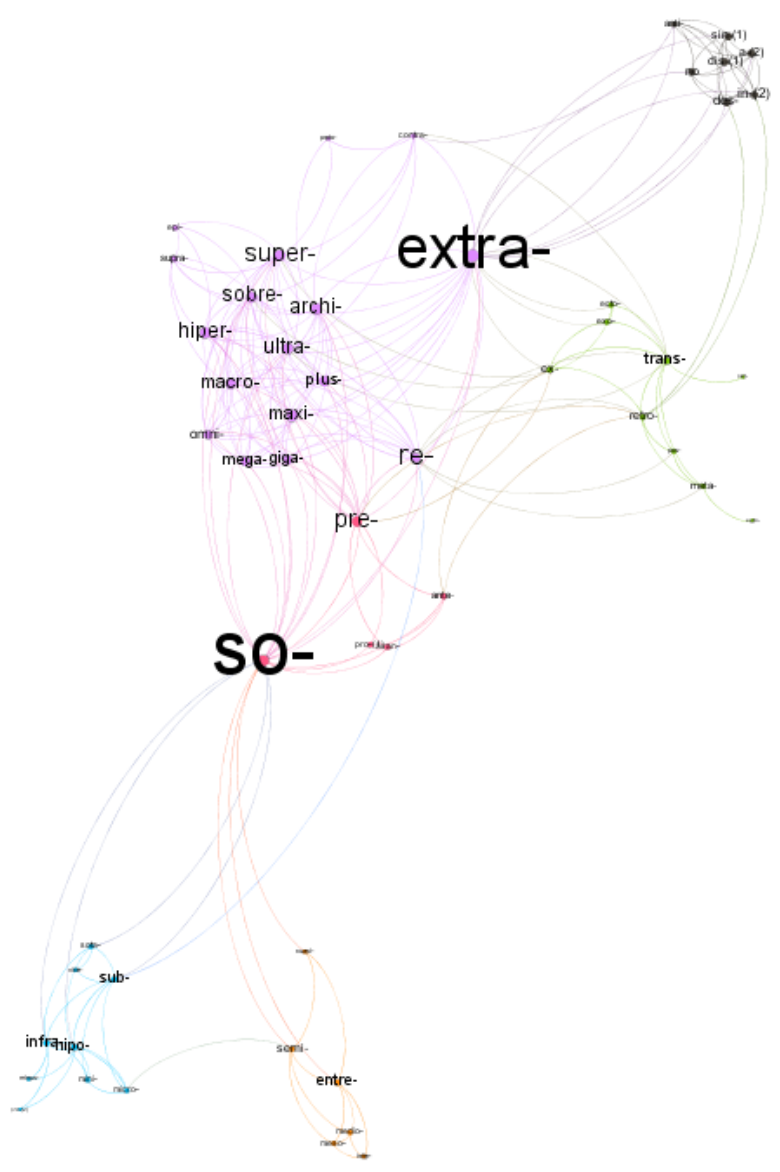

Figura I. Red de la relación entre prefijos a partir de los significados compartidos (El tamaño de los nodos y aristas indica su peso, el color la comunidad y el tamaño de la etiqueta, el grado de intermediación. El grafo tiene una distribución ForceAtlas con una fuerza de repulsión de 20000 y una de atracción de io).

Hesperia. Anuario de filología hispánica XXI-I (20I8) 
De las medidas globales podemos extraer algunas características de la red. En primer lugar, es un grafo poco denso (o, I I 5 ), es decir, se establecen pocas conexiones de las posibles, de hecho, tenemos 7 componentes conexos o siete componentes desconectados entre sí.

A esto se añade que presenta un alto coeficiente medio de clustering $(0,8 \mathrm{O} 2)$ lo cual quiere decir que los prefijos relacionados con un prefijo dado, están también muy relacionados entre sí; así que, con poca densidad, siete componentes conexos y un alto coeficiente de clustering, podemos sospechar que hay grupos de prefijos muy relacionados entre ellos y con pocas o ninguna relación con el resto de grupos y componentes.

Esta organización hace que tenga una modularidad bastante alta $(0,6 \mathrm{O} 2)$ que permite hacer grupos o clústeres (12) de los cuales 6 se encuentran en un gran componente de las 7 que existen y en el que están conectados directa o indirectamente el $80,3 \%$ de los prefijos y los otros 6 se corresponden con los otros 6 componentes desconectados entre sí y en los que se conectan prefijos que están muy marcados léxicamente y son o poco productivos con carácter general o solo productivos para un significado concreto.

- $\mathrm{El} \mathrm{7,58 \%} \mathrm{(dentro-,} \mathrm{intro-,} \mathrm{intra-,} \mathrm{endo-,} \mathrm{ento-).}$

- $\mathrm{El}$ 3,03\% (tele-, apo-).

- $\mathrm{El}$ 3,03\% (circun-, peri-).

- $\mathrm{El}$ 3,03\% (cis-, citra-).

- El I, $52 \%$ (con-).

- El I, $52 \%$ (para-).

Dejaremos ahora de lado estos 6 componentes aislados para centrarnos en el gran componente conexo que podemos ver con más detalle en la Figura 2. De él, además de dar las medidas globales (Tabla 3), prestaremos especial atención a varias de sus medidas (comunidades, cercanía e intermediación). 


\begin{tabular}{|l|r|}
\hline \multicolumn{2}{|c|}{ Medidas globales del subgrafo } \\
\hline $\mathrm{N}$ (nodos-prefijos) & 53 \\
\hline $\mathrm{L}$ (aristas-relaciones) & 233 \\
\hline $\mathrm{d}$ (diámetro) & 5 \\
\hline $\mathrm{D}$ (Densidad) & 0.169 \\
\hline Modularidad & 0.585 \\
\hline Componentes conexos & $\mathrm{I}$ \\
\hline$l_{G}$ (Longitud media del camino) & $2.4 \mathrm{I} 4$ \\
\hline$C$ (Coeficiente medio de clustering) & $0.78^{2}$ \\
\hline
\end{tabular}

Tabla 3: Medidas globales del subgrafo correspondiente al gran componente conexo

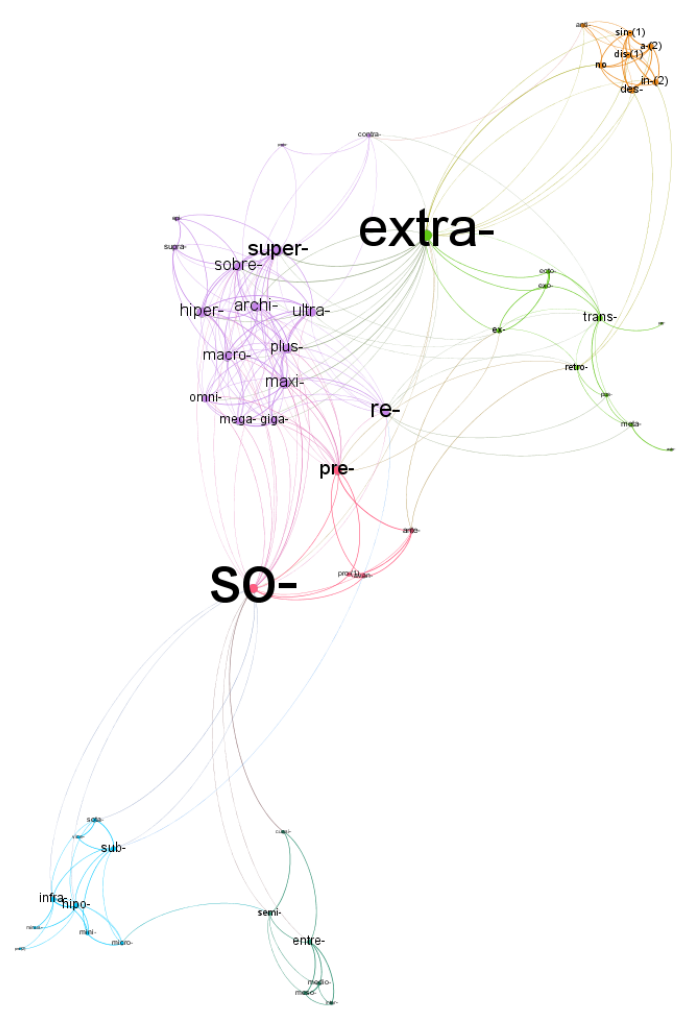

Figura 2. Subgrafo correspondiente al gran componente conexo del grafo de relaciones entre prefijos. (El tamaño de los nodos y aristas indica su peso, el color la comunidad y el tamaño de la etiqueta, el grado de intermediación. El grafo tiene una distribución ForceAtlas con una fuerza de reulsión de 20000 y una de atracción de ro). 
Como se puede ver (Tabla 3) algunas medidas no han variado excesivamente, tenemos un subgrafo poco denso $(0,169)$ con un alto grado de clusterización $\left(0,78^{2}\right)$, lo cual nos remite, igual que antes, a la existencia de grupos de prefijos muy conectados entre sí, pero poco conectados con otros prefijos del subgrafo. Esto hace que todavía se puedan establecer grupos con cierta facilidad, tiene una modularidad de 0,585 frente a la del grafo total que era de 0,602 . Veamos ahora como son esos grupos o clústeres para después tratar las medidas locales de cercanía e intermediación que nos ayudarán a comprender la posición de los prefijos en el grafo.

\section{I. Comunidades}

La división de grafos en comunidades detecta aquellos clústeres de nodos que están más densamente conectados en el grafo, es decir, mantienen más relaciones entre ellos que la media del grafo. En el grafo completo hemos detectado 12 comunidades, de las cuales 6 pertenecen a cada una de los componentes conexos más pequeños y otras 6 se encuentran en el gran componente conexo que hemos separado como subgrafo. Estas últimas 6 comunidades son:

I. Oposición: $a^{-}$, anti-, des-, dis-, in-, no-, sin- (1 $3.21 \%$ de nodos-prefijos).

2. Delante: ante-, avan-, pre-, pro-, so- (9.43\% de nodos-prefijos).

3. Medio: cuasi-, entre-, inter, medio-, meso-, semi- (I I.32\% de nodosprefijos).

4. Detrás-fuera: auto-, ecto-, ex-, exo-, meta-, per-, pos-, retro-, trans- (16.98\% de nodos-prefijos).

5. Arriba-Más: archi-, contra-, epi-, extra-, giga-, hiper-, macro-, maxi-, mega-, omni-, plus-, proto-, re-, sobre-, super-, supra-, ultra- (32.08\% de nodosprefijos).

6. Debajo-Menos: hipo-, infra-, micro-, mini-, minus-, pro-, sota-, sub-, vice( $16.98 \%$ de nodos-prefijos).

Lo primero que hay que indicar es que el nombre dado a cada comunidad es solo un término para facilitar la referencia a él, no es su significado. 
Si bien, como se puede ver, se ha escogido un término que coincidiese en gran parte con el significado principal que indica el conjunto de los prefijos, no quiere decir que no haya más significados en el grupo ni que este sea su único significado. Tal vez sería más adecuado, pues llevaría a menos confusión, etiquetarlos por un número, pero he considerado que esto también dificultaría la comprensión de la explicación.

El hecho de que en una comunidad convivan varios significados conlleva que no todos los prefijos de la comunidad pertenezcan en igual grado a ella ni que se puedan reducir a la etiqueta dada. Por ejemplo, en la comunidad 4. Detrás-fuera, se encuentra el prefijo auto-, cuya clasificación en este grupo parece extraña, pero este prefijo está conectado con el prefijo metapor el significado reflexivo -autoreflexión, metamatemática- y meta-, con trans- y retro- - metatórax, traspatio, retrocarga- y estos dos prefijos conectan indirectamente a auto- con el resto.

Podemos ahora aislar una de esas comunidades para calcular las medidas de ese subgrafo como si se tratase de un grafo; por ejemplo, tomada la comunidad más grande como un grafo.

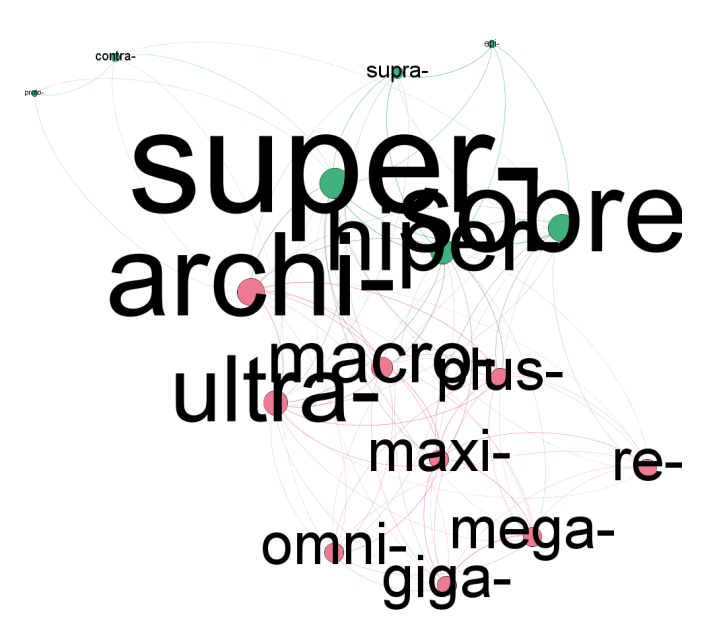

Figura 3. Análisis del subgrafo de la comunidad 5. Arriba-Más como grafo independiente. (El tamaño de los nodos $\mathrm{y}$ aristas indica su grado de centralidad $\left(C_{C L O S}\right.$. El grafo tiene una distribución ForceAtlas con una fuerza de reulsión de 20000 y una de atracción de io). 
En la Figura 3, se puede ver como la comunidad 5. Arriba-Más está dividida en dos comunidades, una en la parte superior (super-, sobre-, hiper-, supra-, contra-, proto-, epi-) y otra, en la inferior, formada por (archi-, macro-, ultra-, plus-, maxi-, omni-, mega-, re-, giga-). Como ya se ha indicado, una comunidad, en estos casos, no representa un significado común, sino una mayor densidad de relaciones de significado que la media del grafo. Esto implica que no sean cortes de significado estrictos; así, que super-pertenezca a una de las comunidades, no implica que no esté relacionado con el resto.

Cada prefijo dentro del grafo y de los subgrafos que establecemos al crear comunidades, tienen distintos grados de relación con el resto de prefijos; para mostrar estos diferentes grados de pertenecia al grafo y a la comunidad, podemos echar mano de una medida de centralidad, la cercanía.

\subsection{Cercanía}

La cercanía (closeness centrality) es una medida local que se corresponde con la longitud media de los caminos más cortos entre un nodo y el resto de nodos del grafo. En la tabla 4 podemos ver las medidas de centralidad de las comunidades 4. Detrás-Fuera y 5. Arriba-Más y del subgrafo completo.

En el apartado anterior habíamos visto como se relacionaba el prefijo auto- con su comunidad, en la Tabla 4, aparecen las medidas de centralidad de la comunidad 4. Detrás-fuera en las que se puede ver que ese prefijo es el menos central de todos. En esas medidas vemos que el prefijo con mayor centralidad es trans-y que tienen una mayor centralidad los prefijos que tienen como significado principal detrás que los que indican fundamentalmente fuera. Llama la atención en esta columna la presencia de extra-, un prefijo que, si repitiésemos la clusterización podría clasificarse en otras comunidades, es decir, es un prefijo a caballo entre varias de ellas, pero este hecho lo explicaremos al tratar la intermediación. 


\begin{tabular}{|l|r|l|l|l|r|}
\hline \multicolumn{7}{|c|}{ Medidas de centralidad $\left(C_{C L O S}\right)$} \\
\hline \multicolumn{7}{|c|}{ Comunidad 4. Detrás-fuera } & \multicolumn{2}{l|}{ Comunidad 5. Arriba-Más } & \multicolumn{2}{l|}{ Subgrafo total (Figura 2) } \\
\hline trans- & 0.89 & super- & I & so- & 0.634 \\
\hline retro- & 0.66 & archi- & 0.937 & extra- & 0.597 \\
\hline meta- & 0.66 & sobre- & 0.937 & re- & 0.577 \\
\hline ex- & 0.62 & hiper- & 0.882 & pre- & 0.547 \\
\hline pos- & 0.62 & ultra- & 0.882 & super- & 0.547 \\
\hline exo- & 0.6 & macro- & 0.789 & sobre- & $0.54 \mathrm{I}$ \\
\hline extra- & 0.6 & giga- & 0.789 & ultra- & $0.54 \mathrm{I}$ \\
\hline ecto- & 0.57 & maxi- & 0.789 & archi- & 0.536 \\
\hline per- & 0.5 & mega- & 0.789 & hiper- & 0.53 \\
\hline auto- & 0.42 & omni- & 0.789 & macro- & 0.525 \\
\hline & & plus- & 0.789 & giga- & 0.52 \\
\hline & & re- & 0.789 & maxi- & 0.52 \\
\hline & & supra- & 0.652 & mega- & 0.52 \\
\hline & & contra- & 0.6 & omni- & 0.52 \\
\hline & & epi- & 0.57 & plus- & 0.52 \\
\hline & & proto- & 0.55 & sub- & 0.45 I \\
\hline
\end{tabular}

Tabla 4. Medidas de centralidad $C_{C L O}$ del subgrafo total (Figura I) y de las comunidades 4. Detrás-fuera y 5 . Arriba-Más (Figura 3) tomadas como subgrafos.

En la segunda columna de la Tabla 4, podemos ver los resultados para la cercanía del subgrafo constituido por la comunidad 5. Arriba-Más. En ella el prefijo central es super-y observamos que, cuanto menos polifuncional sea el prefijo, tiene menor grado de centralidad.

Por último, en la tercera columna, se dan los resultados de cercanía del grafo completo. De estos datos podemos extraer dos conclusiones; una, que el centro del grafo lo ocupan los prefijos pertenecientes a la comunidad 5. Arriba-Más y, la segunda, que, sin embargo, los cuatro primeros prefijos -so-, extra-, re-, pre-- son, en principio, extraños como elementos más centrales. Para explicar el grado de centralidad de estos prefijos hemos de atender a otra medida de centralidad importante en la teoría de redes: la intermediación.

\subsection{Intermediación}

La intermediación (betweenness centrality) $C_{B}$ mide la frecuencia de paso por un nodo de los caminos más cortos entre nodos, también se podría 
decir que cuantifica las veces en que un nodo sirve como puente para unir nodos a través del camino más corto.

Si para las comunidades habíamos dicho que agrupan nodos más densamente conectados que la media, los nodos que sirven para conectar estas comunidades entre ellas, son los nodos con alta intermediación. En nuestro caso, serían aquellos pefijos que mantienen relaciones con otras comunidades y que hacen posible la relación entre ellas debido a que son significados que pueden ser creados por prefijos de ambas comunidades.

En la figura 2, podemos ver como los prefijos so-, extra-, re-, pre- se sitúan como puentes, por decirlo de alguna manera, entre las distintas comunidades; si se eliminasen estos prefijos, las comunidades quedan aisladas o sus enlaces muy debilitados.

Esto nos devuelve a la idea de que los prefijos constituyen una red muy dispersa, poco densa de relaciones, con unos pocos sufijos que sirven de conexión entre las comunidades que conforman la red cuyos nodos-prefijos están muy densamente relacionados.

\section{Conclusiones Y POSIBILIDAdes}

Así que, el grafo de la relación entre prefijos a través de sus significados compartidos es un grafo poco denso con un alto grado de clusterización en el que existen varios componentes aislados y un gran componente que agrupa a los prefijos y significados más productivos que se puede dividir en varios clústeres poco relacionados entre sí pero con unas relaciones internas muy densas que están unidos por unos pocos prefijos con un alto grado de intermediación.

Creo, además, que queda mostrado que el análisis por medio de grafos de las relaciones entre los prefijos a través de sus significados morfológicos puede ser una vía fructífera, que se puede añadir a otros enfoques, para conocer muchos aspectos de la organización morfológica.

Es cierto que todavía queda mucho por perfilar, analizar y mejorar; por ejemplo, en vez de emplear un grafo no dirigido, emplear un grafo dirigido en el que las aristas muestren el diferente grado que hay en la relación dependiendo de la dirección. Pero, tal vez, el gran caballo de batalla sea in- 
troducir en los grafos la frecuencia de creación o la productividad, pues está claro que el peso de una arista no depende solo de los significados compartidos, sino de la frecuencia con que los prefijos compartidos creen uno de los significados relacionados.

Dejo estas ideas para futuros trabajos que espero puedan arrojar un poco más de luz sobre la propuesta hecha aquí y enmendar los errores cometidos en este trabajo exploratorio.

\section{REFERENCIAS BIBLIOGRÁFICAS}

Batyen, H. (2010), "The Directed Compound Graph of English - An Exploration of Lexical Connectivity and its Processing Consequences”. En S. Olsen (Ed.), New Impulses in WordFormation. Buske, 17, 383-402

Bauer, L. (1996), "Derivational paradigms”. En G. E. Booij y J. van Marle (Eds.), Yearbook of Morpbology 1996. Amsterdam: Kluwer Academic Publishers. 243-256.

Beecher, H. (2004), "Derivational Paradigm in Word Formation”. Recuperado de http://citeseerx. ist.psu.edu/viewdoc/summary?doi = Io. I.I.94.907 I

Biemann, C., Choudhury, M., y Mukherjee, A. (2009), "Syntax is from mars while semantics from venus!: insights from spectral analysis of distributional similarity networks". En Proceedings of the ACL-IJCNLP 2009 Conference Short Papers. Association for Computational Linguistics. 245-248.

Blevins, J. P. (200I), Paradigm derivation. Transactions of the Philological Society, 99(2), 2 I I-222.

Bonami, O., y Strnadová, J. (201 8), Paradigm structure and predictability in derivational morphology. Morphology. https://doi.org/ I 0. I o07/s I I 525-0 1 8-9322-6

Ferrer I Cancho, R., y Solé, R. V. (200I), "The small world of human language”. Proc. Biol. Sci., 268(1482), 2261-2265. https://doi.org/Io. 1098/rspb.2001.1 800

Fortunato, S. (20 Io), "Community detection in graphs". Physics Reports, 486:3, 75-174.

Guilbert, L. (1975), La créativité lexicale. París: L’arrousse.

LAhiri, S. (2OI3), Complexity of word collocation networks: A preliminary structural analysis. arXiv Preprint arXiv: 3 I 0.5 I I I.

Marle, J. van. (1985), On the paradigmatic dimension of morphological creativity. Dordrecht: Foris.

MiHALCEA, R., y RADEV, D. (2OI I), Graph-based natural language processing and information retrieval. Cambridge University Press.

Newman, M. E. J. (2010), Networks: an introduction. Oxford; New York: Oxford University Press.

Pena Seijas, J., y Campos Souto, M. (2009), "Propuesta metodológica para el establecimiento de familias léxicas en una consideración histórica: el caso de" hacer." Cuadernos Del Instituto de Historia de La Lengua, 2, 2 I-52.

Plag, I., Andreou, M., y Kawaletz, L. (2017), "A frame-semantic approach to polysemy in affixation". The Lexeme in Descriptive and Theoretical Morphology. Language Science Press: Berlin.

Rainer, F. (1993), Spanische Wortbildungslehre. Tübingen: Niemeyer.

Rainer, F., Dressler, W. U., Gardani, F., y Luschützky, H. C. (20I4), Morphology and meaning: An overview. En F. Rainer, W. U. Dressler, F. Gardani, y H. C. Luschützky (Eds.), Morphology and meaning: selected papers from the is th International Morphology Meeting, Vienna, February 2or2. Amsterdam; Philadelphia: John Benjamins Publishing Company. 3-46.

Real Academia Española (s.f.), Diccionario de la lengua española. Comsultado el i i de Noviembre de 2017 , from http://dle.rae.es 
Real Academia Espanola y Asociacion de Academias de la Lengua Espanola (2009), Nueva gramática de la lengua española (Vol. I). Madrid: Espasa Libros.

RIfón, Antonio (2016), “Estructura de las redes de familias morfológicas antónimas". LEA: Lingüística Española Actual, 38(2), 3 I 5-338.

Rifón, Antonio (2018), "Las redes semánticas de los prefijos en español” en Marta Díaz, Gael Vaamonde, Ana Varela, $\mathrm{M}^{a}$ Carmen Cabeza, José M. García-Miguel e Fernando Ramallo (eds.), Actas do XIII Congreso Internacional de Lingüística Xeral. Vigo: Universidade de Vigo. 775 $782 \mathrm{http}: / /$ cilx 20 I $8 . u v i g o . g a l / a c t a s /$ resumos/66 I $843 . \mathrm{html}$

Štekauer, P. (2O I4), "Derivational paradigms”. En Rochelle Lieber and Pavol Štekauer (eds.),The Oxford Handbook of Derivational Morphology. Oxford: Oxford University Press. 354-369.

Subbotina, N. S., Fatkhutdinova, V. G., y Ratsiburskaya, L. V. (2017), "Derivational paradigmatics in Russian language: linguistic and methodical aspects". Modern Journal of Language Teaching Methods (MJLTM), 7:9/ I, I66- I 7 I.

Varela Ortega, S., y Martín García, J. (i 999), "La prefijación”. En Gramática descriptiva de la lengua española (Vol. 3). Madrid: Espasa Calpe. 4993-5040

recibido: enero de 2018 aceptado: febrero de 2018 